\title{
Activation of cytotoxic $T$ cell population and inversion of CD4:CD8 ratio as manifestations of cellular immune response in SARS-COV-2 infection
}

\author{
Fahad Khan ${ }^{1} \cdot$ Tayler van den Akker ${ }^{1} \cdot$ Shafinaz Hussein ${ }^{1} \cdot$ Bridget K. Marcellino ${ }^{2}$. Pallavi Khattar ${ }^{1}$. \\ Benjamin S. Glicksberg ${ }^{3}$ - Girish N. Nadkarni ${ }^{4}$ - Adolfo Firpo-Betancourt ${ }^{1}$ - Siraj M. El Jamal ${ }^{1}$ (I)
}

Received: 5 June 2020 / Accepted: 23 June 2020 / Published online: 2 July 2020

(C) Springer-Verlag GmbH Germany, part of Springer Nature 2020

To the Editor,

Amid the recent worldwide coronavirus disease 2019 (COVID-19) outbreak caused by the novel severe acute respiratory syndrome coronavirus 2 (SARS-CoV-2), there has been increasing interest in the host-pathogen interaction and the resulting immune dysregulation. The role that the innate immune system plays in responding to SARS-CoV-2 is yet to be fully understood. The effect of this virus on the different lymphocyte populations is based on single-case reports and sporadic series [1-5]. Here, we report the findings from our evaluation of the cellular immune response in six patients infected with SARS-CoV-2 from New York City. We used flow cytometry to analyze helper and cytotoxic $\mathrm{T}$ cell populations, assess the antibody-secreting cells (ASCs) and examine the expression of activation markers.

Briefly, we reviewed flow cytometry studies from six patients who were confirmed positive for the infection by SARS-CoV-2 using nucleic acid testing via RT-PCR of throat swab specimens using RT-PCR. All patients tested positive for SARS-CoV2 within 14 days of the flow cytometry study from our institution, a major tertiary academic center in New York City, which has been a hot spot of the COVID-19 pandemic. Originally, five of

Siraj M. El Jamal

siraj.eljamal@mssm.edu

1 Department of Pathology, Molecular and Cell Based Medicine, Icahn School of Medicine at Mount Sinai, 1 Gustave L. Levy Pl, New York, NY 10029, USA

2 Division of Hematology/Oncology, Icahn School of Medicine at Mount Sinai, Tisch Cancer Institute, New York, NY, USA

3 Department of Genetics and Genomic Sciences, Hasso Plattner Institute for Digital Health at Mount Sinai, New York, USA

4 Department of Nephrology, Icahn School of Medicine at Mount Sinai, New York, NY, USA the flow cytometry studies were performed as a workup for hematological malignancies. Five peripheral blood and one bone marrow aspirate samples are included. Flow cytometry was performed by five-color analysis on the Navios Flow Cytometer (Beckman Coulter, Miami, FL), and data analysis was performed on the Kaluza Flow Cytometry Analysis Software (Beckman Coulter, Miami, FL). Leukocytes were stained with antibodies against CD3, CD4, CD8, CD19, CD38, and HLA-DR. Fluorochromes included fluorescein isothiocyanate (FITC), PEcyanine 5 (PC5), PE-cyanine 7 (PC7), PE-Texas Red (ECD), and phycoerythrin (PE).

Our initial measurements defined both CD4+ and CD8+ T cell populations. We calculated the CD4:CD8 ratio. Then, we assessed the expression of CD38 and HLA-DR on both populations as surrogate markers of activation. To screen for ASCs, we elected to use a simplified two-antibody gating strategy by evaluating the expression of CD38 on the $\mathrm{CD} 19+$ cells taking into account that this phenotype is expressed in most ASCs regardless of their type.

Four out of the six patients included in this study showed varying degrees of lymphopenia (Table 1). Four patients showed characteristically low CD4:CD8 ratio; these four patients showed bright expression of CD38 with partial/dim HLA-DR on the CD8+ T cells indicative of cellular activation (Fig. 1). Analysis of the CD19+ cells for CD38 expression showed no increase in the number of ASCs in any of the patients. The remaining two patients with normal CD4:CD8 ratio showed no expression of CD38 or HLA-DR on the T cells and no evidence of increased ASCs. Although five of the cases had a history of hematologic malignancy, only one case (patient 1) was found to have hematologic neoplasm at the time of the study. For this case, the abnormal population was detected by flow cytometry, whereby this patient had a large B-lymphoblast population (first-time diagnostic study). None of the patients was on treatment for malignancy at the time of study. 
Table 1 Summary of the clinical and laboratory findings of the patients included in the study

\begin{tabular}{cllllll}
\hline Patient & Source & Original diagnosis & $\begin{array}{l}\text { Lymphocytes count } \\
\left.\text { (normal 1.1-4.8 } \times 10^{3} \mu \mathrm{L}\right)\end{array}$ & $\begin{array}{l}\text { CD4:CD8 ratio } \\
\text { (normal 1.0-4.0) }\end{array}$ & $\begin{array}{l}\text { CD38 expression on } \\
\text { cytotoxic T cells }\end{array}$ & $\begin{array}{l}\text { HLA-DR expression on } \\
\text { cytotoxic T cells }\end{array}$ \\
\hline $1^{*}$ & PB & B-ALL & 5.3 & 0.76 & + & + \\
2 & PB & MDS & 0.2 & 4.58 & - & - \\
3 & PB & CNL & 0.3 & 5.82 & - & - \\
4 & PB & AITL & 0.3 & 0.79 & $+(\operatorname{dim})$ & $+(\mathrm{dim})$ \\
5 & BM & PCN & 0.9 & 0.28 & + & + \\
6 & PB & n/a & 3.1 & 0.35 & + & + \\
\hline
\end{tabular}

*Patient \#1 presented with active disease

$B$-ALL B-lymphoblastic leukemia, $M D S$ myelodysplastic syndrome, $C N L$ chronic neutrophilic leukemia, AITL angioimmunoblastic T-cell lymphoma, $P C N$ plasma cell neoplasm, $P B$ peripheral blood, $B M$ bone marrow

In this study, we report some of the characteristics of the cellular immune response to the SARS-CoV-2 virus infection, as seen in six patients in New York City. Our results demonstrate that a subset of SARS-CoV-2-infected patients shows changes in the $\mathrm{T}$ cell population, evident by the decrease in the CD4:CD8 ratio and activation of the cytotoxic T cell population in the setting of lymphopenia. The decrease in the CD4:CD8 ratio is likely due to suppression in the $\mathrm{CD} 4^{+} \mathrm{T}$ cells.

The current observation of lymphopenia is in accordance with the findings from previous reports studying SARSCoV-2 [3, 4]. Lymphopenia is seen in infections by SARS, measles, and H5N1 influenza viruses and in contrast to viruses with lymphocytic proliferative responses like early human immunodeficiency virus (HIV-1), cytomegalovirus (CMV), and Epstein-Barr virus (EBV) [6]. Although the absolute total number of lymphocytes and the absolute lymphocyte subset numbers are decreased, an inverted CD4:CD8 ratio is likely due to the greater reduction of the helper $\mathrm{CD}^{+} \mathrm{T}$ cell population. Moreover, the cytotoxic
$\mathrm{CD} 8^{+} \mathrm{T}$ cell population shows features of activation by expressing CD38 and, to a lesser extent, HLA-DR. These features are identical to what was described in patients with influenza, Ebola, HIV, and CMV infections. The activation of the $\mathrm{CD} 8^{+}$population suggests that similar to the aforementioned viruses, the cytotoxic $\mathrm{T}$ cell population probably plays an integral role in the immune response to SARSCoV-2 infection $[1,3,4,7,8]$. We also speculate that the decrease in the CD4:CD8 ratio and the activation of the cytotoxic $\mathrm{T}$ cells happen synchronously. While proliferation of ASCs would be expected in SARS-CoV-2 patients, we did not detect such an increase in any of our patients. This could be attributed to the possibility that our results provide a snapshot in the natural history of the disease based on a single reading and we do not exclude the possibility of elevated ASC numbers at different time points. Additionally, a small minority of ASCs may not be detected by the simplified CD38 and CD19 panel that we used.

There are several limitations to our study. First, this is a retrospective, single-center study, with a small study sample.

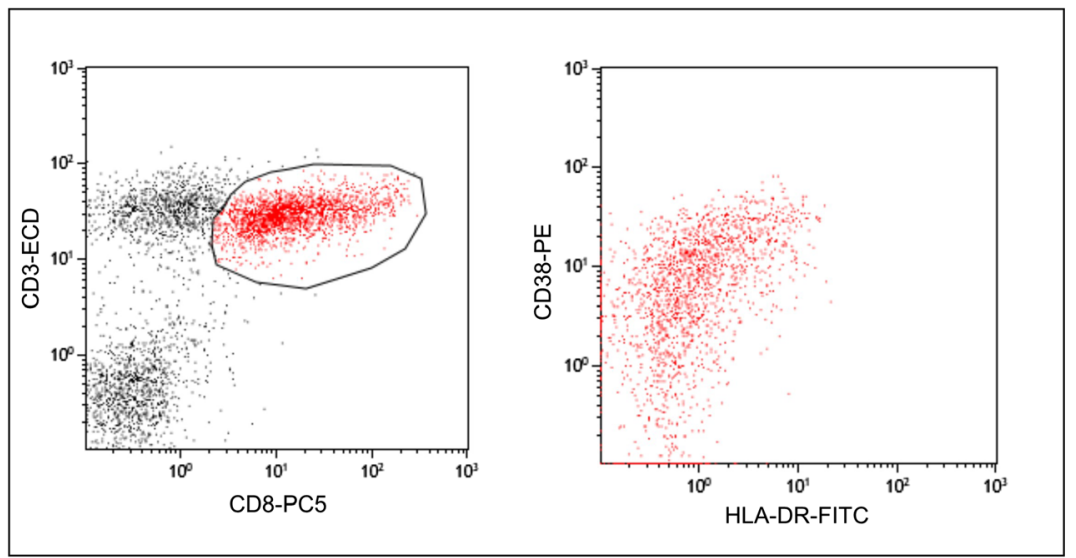

a

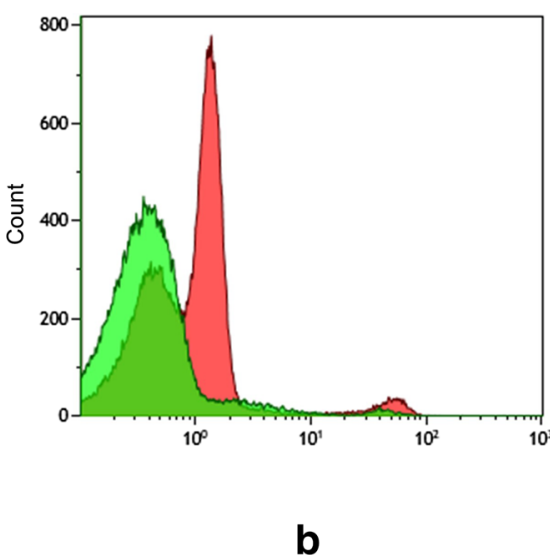

b

Fig. 1 a Flow cytometry analysis of the $\mathrm{T}$ cell population with gating on the $\mathrm{CD} 3^{+} \mathrm{CD} 8^{+} \mathrm{T}$ cells showing bright expression $\mathrm{CD} 38$ with dim/partial expression of HLA-DR. b Mean fluorescence intensity for CD4 and CD8 showing the high number of CD $8^{+}$cells and the low number of CD $4^{+}$cells 
Second, the absence of temporal follow-up evaluations restricts understanding the natural history of the disease and the changes seen in the different studied parameters. Lastly, the underlying hematologic malignancy acting as a confounder is possible as many hematologic malignancies affect the $T$ cell population. However, given that only one case had an active neoplastic disease, and the fact that the changes we report here are not common in hematologic malignancies, it is unlikely that this is actually confounding the results. In fact, hematologic malignancies including, acute leukemia and plasma cell dyscrasia, are associated with a heterogeneous $\mathrm{T}$ cell reaction characterized by decreased levels of naive $\mathrm{CD} 4^{+}$and $\mathrm{CD} 8^{+} \mathrm{T}$ cells and increasing the numbers of the so-called $\mathrm{CD}^{+} / \mathrm{CD} 25^{+}$regulatory $\mathrm{T}$ cells (Tregs) [9]. Despite these limitations, by using flow cytometry, we have confirmed and elaborated on the $\mathrm{T}$ cell compartment alterations found in the setting of SARS-CoV-2 infection $[1-4,10]$.

In conclusion, we document the presence of a distinctive $\mathrm{T}$ cell response in SARS-CoV-2 patients evident by alteration of CD4:CD8 ratio, likely due to suppression of the CD4 population and activation of the cytotoxic $\mathrm{CD} 8^{+} \mathrm{T}$ cell population. Although these findings should be interpreted in the context of the study limitations, they provide potential immune parameters for further detailed studies to elucidate the immune reaction to SARS-CoV-2 virus.

\section{Compliance with ethical standards}

Conflict of interest The authors declare that they have no conflict of interest.

\section{References}

1. Thevarajan I, Nguyen THO, Koutsakos M, Druce J, Caly L, van de Sandt CE, Jia X, Nicholson S, Catton M, Cowie B, Tong SYC, Lewin SR, Kedzierska K (2020) Breadth of concomitant immune responses prior to patient recovery: a case report of non-severe COVID-19. Nat Med 26:453-455

2. Xu Z, Shi L, Wang Y, Zhang J, Huang L, Zhang C, Liu S, Zhao P, Liu H, Zhu L, Tai Y, Bai C, Gao T, Song J, Xia P, Dong J, Zhao J, Wang FS (2020) Pathological findings of COVID-19 associated with acute respiratory distress syndrome. Lancet Respir Med 8: 420-422

3. Wang F, Nie J, Wang H et al (2020) Characteristics of peripheral lymphocyte subset alteration in COVID-19 pneumonia. J Infect Dis

4. Qin C, Zhou L, Hu Z et al (2020) Dysregulation of immune response in patients with COVID-19 in Wuhan. China, Clin Infect Dis

5. El Jamal SM, Salib C, Stock A et al (2020) Atypical lymphocyte morphology in SARS-CoV-2 infection. Pathol Res Pract:153063

6. Li T, Qiu Z, Zhang L, Han Y, He W, Liu Z, Ma X, Fan H, Lu W, Xie J, Wang H, Deng G, Wang A (2004) Significant changes of peripheral $\mathrm{T}$ lymphocyte subsets in patients with severe acute respiratory syndrome. J Infect Dis 189:648-651

7. McElroy AK, Akondy RS, Davis CW et al (2015) Human Ebola virus infection results in substantial immune activation. Proc Natl Acad Sci U S A 112:4719-4724

8. Ganji A, Farahani I, Khansarinejad B, Ghazavi A, Mosayebi G (2020) Increased expression of CD8 marker on T-cells in COVID-19 patients. Blood Cells Mol Dis 83:102437

9. D'Arena G, Vitale C, Coscia M et al (2017) Regulatory T cells and their prognostic relevance in hematologic malignancies. J Immunol Res 2017:1832968

10. Fan BE, Chong VCL, Chan SSW et al (2020) Hematologic parameters in patients with COVID-19 infection. Am J Hematol

Publisher's note Springer Nature remains neutral with regard to jurisdictional claims in published maps and institutional affiliations. 